\title{
P3SONG: Evaluation for autism spectrum disorder
}

\author{
Mandeep Kaur, MD
}

A utism spectrum disorder (ASD) is characterized by impairments in communication and social interactions, along with repetitive and perseverant behaviors. ${ }^{1}$ It has a prevalence of $0.75 \%$ to $1.1 \%$ among the general population. ${ }^{1}$ The presentation of ASD can vary, and patients may have a wide range of comorbidities, such as attention-deficit/hyperactivity disorder (ADHD), neurologic disorders, and genetic disorders. ${ }^{1}$ Therefore, a comprehensive evaluation needs to include a multidisciplinary assessment by clinicians from several specialties, including primary care, psychiatry, psychology, and neurology. Here I offer psychiatrists 3 Ps and the mnemonic SONG to describe a multidisciplinary approach to assessing a patient with suspected or confirmed ASD.

Primary care evaluation of patients with ASD is important for the diagnosis and treatment of any co-existing medical conditions. Primary care physicians are often the source of referrals to psychiatry, although the reason for the referral may not always be suspicion of autism. In my clinical practice, almost all referrals from primary care involve a chief complaint of anger or behavioral problems, or even obsessive-compulsive behaviors.

Psychiatric evaluation should include obtaining a detailed history of the patient's conception, birth, development, and social life, and his/her family history of genetic conditions. In my practice, ADHD and elimination disorders are common comorbidities in patients with ASD. Consider communicating with daycare staff or teachers and auxiliary staff, such as guidance counselors, because doing so can help elucidate the diagnosis. Also, ask adult family members, preferably a parent, for collateral information to help establish an accurate diagnosis in your adult patients.

sychological evaluation should include testing to rule out intellectual disability and learning disorders, which are common in patients with ASD. ${ }^{2}$ Tests commonly used for evaluation of ASD include the Autism Diagnostic Observation Schedule (ADOS), Childhood Autism Rating Scale (CARS), and Autism Diagnostic Interview-Revised (ADI-R).

Speech evaluation. Deficits in language and communication are commonly observed in patients with ASD, especially in younger patients. ${ }^{3}$ A study of the relationship between early language skills (age of first word production) and later functioning in children with ASD indicated that earlier age of first word acquisition was associated with higher cognitive ability and adaptive skills when measured later in childhood. ${ }^{3}$ Therefore, timely intervention following speech evaluation can result in favorable outcomes.

continued

Every issue of CURRENT Psychiatry has its 'Pearls'

Yours could be found here.

Read the 'Pearls' guidelines for manuscript submission at MDedge.com/ CurrentPsychiatry/page/pearls. Then, share with your peers a 'Pearl' of wisdom from your practice.
Dr. Kaur is an Attending General, Child \& Adolescent Psychiatrist, Southeastern Regional Medical Center and affiliated Southeastern Psychiatry Clinic, Lumberton, North Carolina; and Assistant Professor, Department of Psychiatry, Campbell University School of Osteopathic Medicine, Lillington, North Carolina.

Disclosure

The author reports no financial relationships with any companies whose products are mentioned in this article, or with manufacturers of competing products.

doi: $10.12788 / \mathrm{cp} .0073$

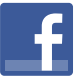

Discuss this article at www.facebook.com/ MDedgePsychiatry 
A baseline EEG

and neuroimaging

can improve your

understanding of

the relationship

between ASD and

seizure disorders
Occupational evaluation. Approximately $69 \%$ to $93 \%$ of children and adults with ASD exhibit sensory symptoms (hyperresponsive, hyporesponsive, and sensoryseeking behaviors). ${ }^{4}$ Patients with sensory symptoms often experience limitations in multiple areas of their life. Early intervention by an occupational therapist can help improve long-term outcomes. ${ }^{4}$

Neurologic evaluation is important because ASD is a neurodevelopmental disorder. Patients with ASD often have comorbid seizure disorders. ${ }^{1}$ The estimated prevalence of epilepsy in these patients ranges from $2.7 \%$ to $44.4 \% .{ }^{1} \mathrm{~A}$ baseline EEG and neuroimaging can help improve your understanding of the relationship between ASD and seizure disorders, and guide treatment.

Genetic testing. Between $10 \%$ to $15 \%$ of individuals with ASD have a medical condition, such as cytogenetic or single-gene disorder, that causes ASD. ${ }^{5}$ Fragile $X$ syndrome, tuberous sclerosis, and Prader-Willi syndrome are a few common examples of genetic disorders associated with ASD. 5 Autism spectrum disorder has also been known to have a strong genetic basis with high probability of heritability in families. ${ }^{5}$ Genetic testing can help to detect any underlying genetic disorders in your patients as well as their family members. Chromosomal microarray analysis has become more accessible due to improved insurance coverage, and is convenient to perform by collection of a buccal mucosa sample in the office setting.

\section{References}

1. Strasser L, Downes M, Kung I, et al. Prevalence and risk factors for autism spectrum disorder in epilepsy: a systematic review and meta-analysis. Dev Med Child Neurol. 2018;60(1):19-29.

2. Schwatrz CE, Neri G. Autism and intellectual disability: two sides of the same coin. Am J Med Genet Part C Semin Med Genet. 2012;160C(2):89-89.

3. Mayo J, Chlebowski C, Fein DA, et al. Age of first words predicts cognitive ability and adaptive skills in children with ASD. J Autism Dev Disord. 2013;43(2): 253-264.

4. McCormick C, Hepburn S, Young GS, et al. Sensory symptoms in children with autism spectrum disorder, other developmental disorders and typical development: a longitudinal study. Autism. 2016;20(5):572-579.

5. Balasubramanian B, Bhatt CV, Goyel NA. Genetic studies in children with intellectual disability and autistic spectrum of disorders. Indian J Hum Genet. 2009;15(3): 103-107. 